\title{
Effects of nanoscale size dependent parameters on lattice thermal conductivity in Si nanowire
}

\author{
M S OMAR and H T TAHA \\ Department of Physics, College of Science, University of Salahaddin, Arbil, Iraqi \\ Kurdistan, Iraq \\ e-mail: dr_m_s_omar@yahoo.com
}

MS received 4 August 2008; revised 16 October 2009; accepted 15 January 2010

\begin{abstract}
The effects of nanoscale size dependent parameters on lattice thermal conductivity are calculated using the Debye-Callaway model including transverse and longitudinal modes explicitly for Si nanowire with diameters of 115, 56, 37 and $22 \mathrm{~nm}$. A direct method is used to calculate the group velocity for different size nanowire from their related calculated melting point. For all diameters considered, the effects of surface roughness, defects and transverse and longitudinal Gruneisen parameters are successfully used to correlate the calculated values of lattice thermal conductivity to that of the reported experimental curve. The obtained fitting value for mean Gruneisen parameter has a systematic dependence on all Si nanowire diameters changing from 0.791 for $115 \mathrm{~nm}$ diameter to 1.515 for the $22 \mathrm{~nm}$ nanowire diameter. The dependence also gave a suggested surface thickness of about $5-6 \mathrm{~nm}$. The other two parameters were found to have partially systematic dependence for diameters 115,56 , and $37 \mathrm{~nm}$ for defects and 56, 37 and $22 \mathrm{~nm}$ for the roughness. When the diameters go down from 115 to $22 \mathrm{~nm}$, the concentration of dislocation increased from $1.16 \times 10^{19} \mathrm{~cm}^{-3}$ to $5.20 \times 10^{19} \mathrm{~cm}^{-3}$ while the surface roughness $P$ found to increase from 0.475 to 0.130 and the rms height deviation from the surface changes by about 1.66 in this range of diameter. The diameter dependence also indicates a strong control of surface effect in surface to bulk ratio for the $22 \mathrm{~nm}$ wire diameter.
\end{abstract}

Keywords. Si nanowire; lattice thermal conductivity; Gruneisen parameter; lattice defect; surface roughness.

\section{Introduction}

Determination of thermal conductivity of semiconductor nanowires plays a crucial role in the development of a new generation of thermoelectric materials (Mahan et al 1997). First measurements of thermal conductivity for $\mathrm{Si}$ nanowires have been reported by ( $\mathrm{Li}$ et al 2003). From theoretical point of view, it is important to be able to, quantitatively calculate lattice thermal conductivity of nanowires in a predictive fashion. In the past several decades, techniques for calculations of lattice thermal conductivity have become wide spread for bulk 
materials (Callaway 1959; Holand 1963). The works have been based on the linearised dispersion models that involve a certain number of adjustable parameters. By taking a proper account of the boundary scattering mechanism, researchers tried unsuccessfully to predict the lattice thermal conductivity of nanowires compared to that of the bulk. Recently, Murphy \& Moore (2007) studied the effect of coherence between different scattering events in quasi-onedimensional systems of variable transverse dimension using a large scale numerical transverse matrix approach to check a simple analytic model. In general, some authors were successful in calculating lattice thermal conductivity for Si nanowires for the diameters of 115, 56 and $37 \mathrm{~nm}$ but none of them succeeded to obtain an accurate value for the $22 \mathrm{~nm}$ diameter which can agree with experimental curve (Li 2002; Mingo 2003).

The decrease of a nanowire lattice thermal conductivity at low temperature is due to scattering from both the rough surface and modified structure defects. Rough surfaces are known to decrease thermal conductance below the usual level (Chen et al 2005; Santamore \& Cross 2001). An obvious reduction of lattice thermal conductance at sufficiently low temperature has been observed (Lu et al 2002). Investigation on the effect of defects on lattice thermal conductance in the nanowire structure also showed the decrease of thermal conductance at low temperature (Chen et al 2005).

It is well-known that, when the size of a bulk material reduces to the range of nanoscale, its structural properties become similar to that of the surface. In such a case, different unit cells are expected for different size and shape of the surface crystalline layers. When the crystal layers are bent to a cylindrical shape forming a nanowire or from 2D (two-dimensional) to that of 1D (one-dimensional) lattice orientation, a new shape of lattice deformation will be created. This deforms the surface structure and that creates extra modes in the lattice vibration which consequently can be represented by the Gruneisen parameter. Such a bend model in lattice arrangements also acts as a lattice dislocation, which is the case for nanowire size materials. Depending on this line of thought, the decrease of nanowire size should also represent an increase of lattice dislocation.

Depending on the above information, the effects of modification on Gruneisen parameter, surface roughness and lattice dislocation on the lattice thermal conductivity in Si nanowire are investigated in this work.

\section{Calculation of lattice thermal conductivity}

It is well-known that, the lattice thermal conductivity is represented by

$$
K_{t h}=\frac{1}{3} \sum C_{v}(\omega) v_{g} \lambda(\omega)
$$

$C_{v}(\omega)$ is the contribution to the specific heat per frequency interval from phonon of frequency $\omega, \lambda(\omega)$ is the phonon mean free path, $v_{g}$ is the phonon group velocity.

Following the approach used by (Asen-Palmer et al 1997) on Eq. (1), the lattice thermal conductivity was calculated through the sum over one longitudinal $\left(K_{L}\right)$ and two degenerate transverse $\left(K_{T}\right)$ phonon branches:

$$
K=K_{L}+2 K_{T}
$$

where

$$
K_{L}=K_{L_{1}}+K_{L_{2}}
$$


The partial conductivities $K_{L_{1}}$ and $K_{L_{2}}$ are the usual Debye-Callaway terms given by:

$$
\begin{aligned}
K_{L_{1}} & =\frac{1}{3} A_{L} T^{3} \int_{0}^{\theta_{L} / T} \frac{\tau_{C}^{L}(x) x^{4} e^{x}}{\tau_{N}^{L}(x)\left(e^{x}-1\right)^{2}} d x \\
K_{L_{2}} & =\frac{1}{3} A_{L} T^{3} \frac{\left[\int_{0}^{\theta_{L} / T} \frac{\tau_{C}^{L}(x) x^{4} e^{x}}{\tau_{N}^{L}(x)\left(e^{x}-1\right)^{2}} d x\right]^{2}}{\int_{0}^{\theta_{L} / T} \frac{\tau_{C}^{L}(x) x^{4} e^{x}}{\tau_{N}^{L} \tau_{R}^{L}(x)\left(e^{x}-1\right)^{2}} d x}
\end{aligned}
$$

and similarly, for the transverse phonons,

$$
\begin{aligned}
& K_{T_{1}}=\frac{1}{3} A_{T} T^{3} \int_{0}^{\theta_{L} / T} \frac{\tau_{C}^{T}(x) x^{4} e^{x}}{\tau_{N}^{T}(x)\left(e^{x}-1\right)^{2}} d x \\
& K_{T_{2}}=\frac{1}{3} A_{T} T^{3} \frac{\left[\int_{0}^{\theta_{L} / T} \frac{\tau_{C}^{T}(x) x^{4} e^{x}}{\tau_{N}^{T}(x)\left(e^{x}-1\right)^{2}} d x\right]^{2}}{\int_{0}^{\theta_{L} / T} \frac{\tau_{C}^{T}(x) x^{4} e^{x}}{\tau_{N}^{T} \tau_{R}^{T}(x)\left(e^{x}-1\right)^{2}} d x} .
\end{aligned}
$$

In these expressions, $\left(\tau_{N}\right)^{-1}$ is the scattering rate for normal phonon processes, $\left(\tau_{R}\right)^{-1}$ is the sum of all resistive scattering processes, and $\left(\tau_{C}\right)^{-1}=\left(\tau_{N}\right)^{-1}+\left(\tau_{R}\right)^{-1}$, with superscripts $L$ and $T$ denoting longitudinal and transverse phonons, respectively. The quantities $\theta_{L}$ and $\theta_{T}$ are Debye temperatures appropriate for the longitudinal and transverse phonon branches, respectively, and

$$
A_{L(T)}=\frac{k_{B}^{4}}{2 \pi^{2} \hbar^{3} v_{L(T)}}
$$

and

$$
x=\frac{\hbar \omega}{k_{B} T},
$$

here $\hbar$ is the Planck constant divided by $2 \pi, k_{B}$ is the Boltzmann constant, $\omega$ is the phonon frequency, and $v_{L(T)}$ are the longitudinal (transverse) acoustic phonon velocities, respectively.

The temperature dependence and the magnitude of the lattice thermal conductivity calculated using the Callaway model are dependent on the temperature and frequency dependence of the scattering rates comprising $\left(\tau_{N}\right)^{-1}$ and $\left(\tau_{R}\right)^{-1}$, their coefficients, Debye temperatures and phonon velocities.

In this model, the acoustic phonon relaxation is considered as a resistive process, they include unharmonic interaction (three-phonon Umklapp scattering, $\tau_{U}$ ), mass difference scattering (isotopes and impurity, $\tau_{M}$ ), boundary scattering $\tau_{B}$ and $\tau_{N}$ normal three phonon scattering. According to Matthiessen's rule, the combination of relaxation times has the form:

$$
\frac{1}{\tau_{C}}=\frac{1}{\tau_{U}}+\frac{1}{\tau_{M}}+\frac{1}{\tau_{B}}+\frac{1}{\tau_{N}}
$$

The phonon-phonon umklapp scattering rate $\tau_{U}$ for phonons of velocity $v$ and Gruneisen parameter $\gamma$ has been suggested by (Slack \& Galginaitis 1964) in the following form:

$$
\tau_{U}^{-1}(\omega)=B_{U} \omega^{2} T e^{-\theta_{D} / 3 T},
$$


with

$$
B_{U} \approx \frac{\hbar \gamma^{2}}{M v^{2} \theta_{D}},
$$

where $M$ is the average mass of an atom in the crystal, and $\gamma$ is the Gruneisen unharmonicity parameter. Then, the umklapp scattering rate for longitudinal and transverse phonons are fixed as (replacing $\omega$ with $x$ ), (Slack \& Galginaitis 1964)

$$
\left[\tau_{U}^{L}(x)\right]^{-1}=B_{U}^{L}\left(\frac{k_{B}}{\hbar}\right)^{2} x^{2} T^{3} e^{-\theta_{D}^{L} / 3 T}
$$

with

$$
B_{U}^{L}=\frac{\hbar \gamma_{L}^{2}}{M v_{L}^{2} \theta_{D}^{L}}
$$

and

$$
\left[\tau_{U}^{T}(x)\right]^{-1}=B_{U}^{T}\left(\frac{k_{B}}{\hbar}\right)^{2} x^{2} T^{3} e^{-\theta_{D}^{T} / 3 T}
$$

with

$$
B_{U}^{T}=\frac{\hbar \gamma_{T}^{2}}{M v_{T}^{2} \theta_{D}^{T}} .
$$

The umklapp scattering rate thus depends on the transverse and longitudinal Debye temperature, phonon velocities, and Gruneisen parameters and, as such, will be different for different phonon modes.

Since it is assumed that heat is carried only by acoustic phonons, the parameters corresponding to these modes will only be considered. However, values of Debye temperature calculated from specific heat at low temperature is the average of all phonon modes, both acoustic and optic, and would be too high an estimate for the acoustic modes only. The other approach was given by (Morelli et al 2002) to calculate the Debye temperature for each acoustic branch from acoustic mode phonon velocity. This also yields an over estimate of Debye temperature because it neglects dispersion of the phonon branches near the zone boundary (Morelli et al 2002). For lattice thermal conductivity, were the heat assumed to be carried only by acoustic phonons, phonon frequencies only up to but not exceeding the maximum frequency $w_{\max }$ at the zone boundary is to be considered. In this work, Debye temperature values used are $240 \mathrm{~K}$ and $586 \mathrm{~K}$ for transverse and longitudinal when they have been calculated from the zone boundary frequencies (Dolling \& Cowley 1966).

The mode of Gruneisen parameter for Si evolve from positive to negative as the phonon wave vector moves out word from the zone center to the zone edge (Xu et al 1991). The longitudinal modes have $\gamma$ in the range of $0.9-1.3$ independent of frequency whereas the transverse modes along $\{100\}$ are in the range of $0.3-1.0$ for low frequency. The model used in this work for lattice thermal conductivity, needs the longitudinal and transverse phonon Gruneisen parameter are to be used as adjustable parameter in the range of values mentioned above (Morelli et al 2002). The best fit values for both longitudinal and transverse Gruneisen parameter were found in this work to be 1.08 and 0.58 respectively. 
Mass-difference scattering arises due to the presence of atoms with a mass difference from that of the average atomic mass in a semiconductor. However, different mass can come from the isotopes of a particular element or impurity atoms. The relaxation rate for the massdifference scattering is calculated from the relation:

$$
\frac{1}{\tau_{M}}=A \omega^{4}
$$

where $\omega$ is the angular frequency $\left(\omega=\frac{k_{B}^{T}}{\hbar} x\right)$, and $A$ is the impurity strength parameter, which at least is the sum of two terms $A=A_{\text {iso }}+A_{\text {imp }}$, where $A_{\text {iso }}$ being due to the scattering by distribution of isotopes of the elements in the compound which can be calculated from:

$$
A_{\text {iso }}=\frac{V_{o}}{4 \pi v_{g}^{3}} \Gamma_{M}
$$

where $V_{o}$ is the lattice volume and $\Gamma_{M}$ is the measure of the strength of the mass-difference scattering defined as

$$
\Gamma_{M}=\sum_{i} f_{i}\left(1-\frac{M_{i}}{\bar{M}}\right)^{2} .
$$

Here, $f_{i}$ is the fractional concentration of the impurity atoms of mass $M_{i}$ and $\bar{M}=\sum_{i} f_{i} M_{i}$ is the average atomic mass and is equal to $(28.0855 \mathrm{amu})$ for Si. Silicon isotopes are $92.2 \%$ ${ }^{28} \mathrm{Si}, 4.7 \%{ }^{29} \mathrm{Si}$ and $3 \cdot 1 \%{ }^{30} \mathrm{Si}$. Then according to Eq. (17), value of $\Gamma_{M}$ was found to be equal to $2 \cdot 005 \times 10^{-4}$. While $A_{\text {imp }}$ is the strength parameter due to scattering caused by impurities and is given by the relation:

$$
A_{\text {imp }}=\frac{3 V_{o}^{2} S^{2}}{\hbar v_{g}^{3}} N_{\mathrm{imp}},
$$

where $S$ is the scattering factor which usually has a value close to unity (Klemens 1955) and $N_{\text {imp }}$ is the impurity concentration. However, impurity concentration up to $10^{17} \mathrm{~cm}^{-3}$ has no effect on lattice thermal conductivity in bulk crystals (Slack \& Galginaitis 1964).

The phonon-boundary scattering rate is assumed independent of temperature and frequency, and can be written as

$$
\left(\tau_{B}^{L}\right)^{-1}=\frac{v_{L}}{d}
$$

and

$$
\left(\tau_{B}^{T}\right)^{-1}=\frac{v_{T}}{d}
$$

where $d$ is the effective diameter of the sample. The value of $d$ will be adjusted slightly in order to fit the $T^{3}$ dependence of the thermal conductivity at the lowest temperatures. For the experimental data used in this work as shown in figure 1 , the value of $d$ found to be equal to $5 \mathrm{~mm}$.

Although normal phonon scattering is not a resistive process, the Callaway model requires knowledge of this scattering rate in order to calculate thermal conductivity. (Herring 1954) has suggested several forms depending on the crystal class. The approach of 


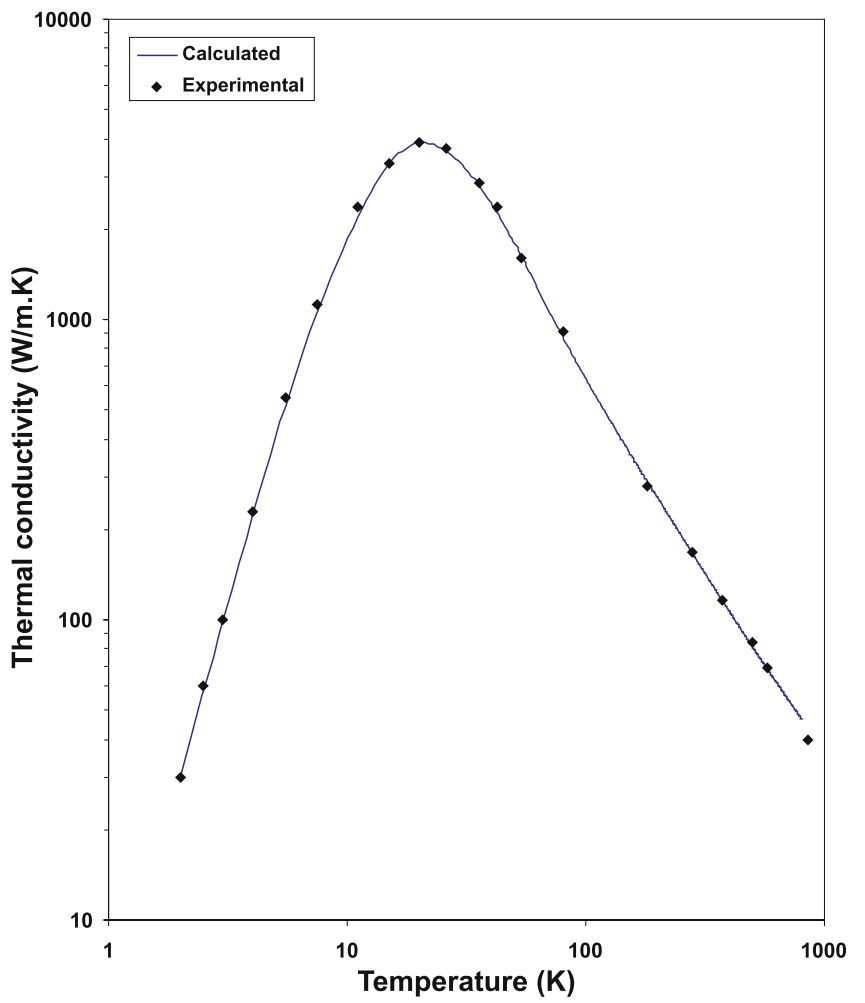

Figure 1. Temperature dependence of the calculated lattice thermal conductivity fitted to the experimental data taken from Morelli et al (2002), for bulk pure single crystal of $\mathrm{Si}$.

(Asen-Palmer et al 1997) which has been used by (Morelli et al 2002) for Si is also used for the present work. The appropriate forms for longitudinal and transverse phonons are

$$
\left[\tau_{N}^{L}(\omega)\right]^{-1}=B_{N}^{L} \omega^{2} T^{3}
$$

and

$$
\left[\tau_{N}^{T}(\omega)\right]^{-1}=B_{N}^{T} \omega T^{4},
$$

or in terms of the dimensionless variable $x$,

$$
\left[\tau_{N}^{L}(\omega)\right]^{-1}=B_{N}^{L}\left(\frac{k_{B}}{\hbar}\right)^{2} x^{2} T^{5}
$$

and

$$
\left[\tau_{N}^{T}(\omega)\right]^{-1}=B_{N}^{T}\left(\frac{k_{B}}{\hbar}\right) x T^{5} .
$$

The magnitudes $B_{N}$ of the normal phonon-scattering rates are reported to be used as adjustable parameters without regarding to their dependence on the properties of a given crystal and the general formula for phonon scattering rate coefficient is expressed as (Morelli et al 2002),

$$
B_{N}(a, b) \approx\left(\frac{k_{B}}{\hbar}\right)^{b} \frac{\hbar \gamma^{2} V^{(a+b-2) / 3}}{M v^{a+b}},
$$


where $a$ and $b$ are constant adjustable parameters. Equation (24) is similar to umklapp processes, Eq. (13), except that the dependence on Debye temperature is replaced by dependence on phonon velocity. This reflects the fact that normal processes involve mostly phonons with low wave vector for which dispersion at the zone boundary is unimportant. In this work, the cases $(a, b)$ as $(1,4)$ and $(a, b)$ as $(2,3)$ for longitudinal and transverse phonon scattering respectively are considered as follows (Ositinskaya 1992):

$$
B_{N}^{L}=\frac{k_{B}^{3} \gamma_{L}^{2} V_{o}}{M \hbar^{2} v_{L}^{5}}
$$

and

$$
B_{N}^{T}=\frac{k_{B}^{4} \gamma_{T}^{2} V_{o}}{M \hbar^{3} v_{T}^{5}} .
$$

Calculations through equations from (3) to (26) and their substitutional in Eq. (2) gave values of lattice thermal conductivity for bulk $\mathrm{Si}$ as shown in figure 1 with that of the reported experimental values.

In order to calculate lattice thermal conductivity for Si nanowires, the crystal size dependent parameters should be taken care of. These parameters are $v_{g}, \theta_{D}, P$ (surface roughness), $\gamma$ and lattice dislocation which is regarded as a defect impurity $N_{\text {imp. }}$.

As mentioned above, the phonon group velocity $v_{g}$ is a function of the materials size and that to for a low-dimensional structure it depends on a particular type of boundaries (Balandin \& Wang 1998).

When the system considered is assumed to be isotropic, the average group velocity is proportional to the crystals characteristic Debye temperature (Post 1953; Regel \& Glazov 1995). For this case it will be (Liang \& Baowen Li 2006);

$$
\theta_{D} \propto \frac{2 h}{\pi k_{B}}\left(\frac{3 N_{A}}{4 \pi V}\right)^{1 / 3} v_{g},
$$

where $h$ is Plank constant, $k_{B}$ is Boltzmann constant, $N_{A}$ is Avogadro number, and $V$ is the volume per atom. Equation (27) is assumed to be valid for the nanoscale size crystals and if $L$ is the size dimension of nanostructure, such as the diameter of nanowires or the thickness of thin films, and the subscript $B^{\prime}$ for $(D \rightarrow \infty)$ be the corresponding bulk limit, then Eq. (27) for both the bulk and nanowire will give the size dependence of both the phonon group velocity and the Debye temperature in the form;

$$
\frac{v_{g}^{n}}{v_{g}^{B^{\prime}}}=\frac{\theta_{D}^{n}}{\theta_{D}^{B^{\prime}}},
$$

where $v_{g}^{B^{\prime}}$ is bulk group velocity, $v_{g}^{n}$ is the nanowire group velocity, $\theta_{D}^{B^{\prime}}$ is bulk Debye temperature and $\theta_{D}^{n}$ is the nanowire Debye temperature.

Lindemanns proposition of melting criterion, known to be valid for small particles, which states that a crystal melts when the root mean square displacement (MSD) of atoms in the crystal exceeds a certain fraction of the inter-atomic distance (Lindemann 1910). From this, the relationship between the melting point and the Debye temperature of crystals can be determined. From the Einstein specific heat theory, the square of the characteristic temperature 
Table 1. The diameter dependence calculated values for $v_{g}, \theta_{D}$ and $T_{m}$ by using Eqs. (27, 29 and 30).

\begin{tabular}{|c|c|c|c|c|c|}
\hline \multirow{2}{*}{$\begin{array}{l}\text { Diameter } \\
(\mathrm{nm})\end{array}$} & \multirow{2}{*}{$\begin{array}{c}\text { Melting point } \\
T_{m}(\mathrm{~K})\end{array}$} & \multicolumn{2}{|c|}{$\begin{array}{c}\text { Debye temperature } \\
\theta_{D}(\mathrm{~K})\end{array}$} & \multicolumn{2}{|c|}{$\begin{array}{c}\text { Group velocity } \\
v_{g} \times 10^{3}(\mathrm{~m} / \mathrm{sec})\end{array}$} \\
\hline & & $\theta_{D}^{L}$ & $\theta_{D}^{T}$ & $v_{g}^{L}$ & $v_{g}^{L}$ \\
\hline Bulk & 1690 & 586 & 240 & 8.47800 & $5 \cdot 850$ \\
\hline 115 & 1658 & $580 \cdot 041$ & 237.560 & 8.39179 & 5.791 \\
\hline 56 & 1620 & 573.678 & 234.954 & 8.29974 & $5 \cdot 727$ \\
\hline 37 & 1583 & $567 \cdot 220$ & $232 \cdot 309$ & 8.20630 & $5 \cdot 663$ \\
\hline 22 & 1510 & 553.967 & $226 \cdot 881$ & 8.01456 & $5 \cdot 530$ \\
\hline
\end{tabular}

is proportional to the melting point $T_{m}$ of crystals as follows (Dash 1999);

$$
\theta_{D}=\text { Const. }\left(\frac{T_{m}}{M V^{2 / 3}}\right)^{1 / 2}
$$

where $M$ is the molecular mass. By dividing the left and right hand side of Eq. (29) applicable to a nanowire and bulk, the proportional relation gives (Liang \& Baowen Li 2006),

$$
\frac{\left(\theta_{D}^{n}\right)^{2}}{\left(\theta_{D}^{B^{\prime}}\right)^{2}}=\frac{T_{m}^{n}}{T_{m}^{B^{\prime}}},
$$

where $T_{m}^{B^{\prime}}$ is the melting temperature for the bulk and for $\mathrm{Si}$ is equal to $1685 \mathrm{~K}$ and $T_{m}^{n}$ for a nanowire. In the nanoscale range, relation (30) gives a size dependent Debye temperature $\theta_{D}^{n}$ calculated from the samples melting point. The size dependent melting point is calculated from the relation (Zhang et al 2001):

$$
\frac{T_{m}^{n}}{T_{m}^{B^{\prime}}}=\exp \left(-\frac{2\left(S_{m}-R\right)}{3 R\left(r_{n} / r_{c}-1\right)}\right),
$$

where $S_{m}$ is the bulk overall melting entropy and $R$ is the ideal gas constant, and for Si they are equal to $30 \mathrm{~J} \mathrm{~mol}^{-1} \mathrm{~K}^{-1}$ and $8 \cdot 31 \mathrm{~J} \mathrm{~mol}^{-1} \mathrm{~K}^{-1}$, respectively (Lindemann 1910 ), $r_{c}$ denotes a critical radius at which all atoms of the particle are located on the bulk surface and $r_{n}$ is the nanowire radius. For low-dimensional crystals, it is clear that $r_{c}$ in Eq. (31) depends on the structure dimension $d$ : where $d=0$ for a nanocrystal, 1 for nanowires and 2 for thin films (Zhang et al 2001). For a nanoparticle, $r_{n}$ has the usual meaning of radius. For a nanowire, $r_{n}$ is taken to be its radius. For a thin film, $r_{n}$ denotes its half thickness. In short, the relationship between $d$ and $r_{c}$ is given by (Wen et al 2000),

$$
r_{c}=(3-d) a_{o}
$$

where $a_{o}$ is the diameter of a spherical volume occupied by a crystal lattice and its value is equal to $0.3338 \mathrm{~nm}$ for $\mathrm{Si}$ (Zhang et al 2001). Values for $T_{m}^{B^{\prime}}, \theta_{D}^{n}$ and $v_{g}^{B^{\prime}}$ calculated through equations from 28 to 32 are given in table 1 , and they are used in the present calculation.

For pure crystals in the range of liquid helium $(T<10 \mathrm{~K})$, lattice thermal conductivity is limited by scattering of phonons at the crystal surface which is called boundary scattering 
(Wen et al 2000). In this region, thermal conductivity is proportional to $T^{3}$ and depends linearly on the sample dimensions when the surface scattering is strictly diffuse (Wen et al 2000: Casimir 1938). In such cases, Eq. (1) for thermal conductivity approaches $K=\frac{1}{3} C_{V} v_{B} L_{C}$, where $L_{C}$ is the so-called 'Casimir length'. For a circular cylinder with radius $R$, this length is equal to $L_{C}=2 R$ while for a square or rectangular cross-section with side lengths $a$ and $b$, $L_{C}=1 \cdot 12 L_{G}$, and $L_{G}=(a b)^{0 \cdot 5}$. The relevant relaxation rate as a cylindrical sample size with radius $R$ becomes [in analogy to Eq. (18)]:

$$
\frac{1}{\tau_{B}}=\frac{v_{L(T)}}{L_{E}}=\frac{v_{L(T)}}{2 R}
$$

There is a finite sample length effect resulting in a decrease of $K$ which can be approximated by defining an effective mean free path $\frac{1}{L_{E}}=\frac{1}{L_{C}}+\frac{1}{l}$, where $l$ denotes the length of the sample in the direction of the heat flow (Berman 1976; Vandersande 1977) which is $4 \mu$ (Li 2002). In this case the relaxation rate for boundary scattering $\tau_{B}^{-1}$ can be written in a general form, including the size correction discussed before:

$$
\frac{1}{\tau_{B}}(l)=\frac{v_{i}}{L_{E}}=v_{L(T)}\left(\frac{1}{L}+\frac{1}{l}\right) .
$$

There is also Partial specularity of the phonon scattering at the sample surfaces which can decrease the effect of boundary scattering. The sample then appears to have larger dimensions than it actually has (Berman et al 1953; Thacher 1967). The relaxation rate is then rewritten in the form

$$
\frac{1}{\tau_{B}}(l, P)=v_{L(T)}\left(\frac{1}{L_{C}} \frac{(1-P)}{(1+P)}+\frac{1}{l}\right) .
$$

The expression for the effective mean free path $L_{E}$ (or the relaxation rate $\tau_{B}^{-1}$ ) used for the description of the $K(T)$ curves may encompass various effects and has the form

$$
L_{E}(l, P)=\left(\frac{1}{L_{C}} \frac{(1-P)}{(1+P)}+\frac{1}{l}\right)^{-1} .
$$

In principle, Eq. (36) enables us to calculate $P$ from the sample geometry (Casimir length) and from the effective mean free path $L_{E}$, determined from the low temperature range in which $K(T)$ has the dependence of $T^{3}$ and then $P$ varies from zero to one. Whenever $P>0$, partial specular reflection occurs (e.g. $P=0.5$ corresponds to a phonon mean free path of three times the geometrical Casimir length with an average of two boundary reflections; $P=0.75$ yields (seven-fold length and six-fold reflections)). It has been found that, the determination of $P$ using Eq. (36) is hampered by the following (Slack \& Galginaitis 1964): (a) the temperature range of validity of the $T^{3}$ law is rather limited in most samples studied, (b) the influence of isotopic scattering extends down to the lowest temperatures. Hence, the parameters $P, \gamma$ and $N_{\text {imp }}$ have been determined by a variation method accounting for boundary and isotopic scattering. For this purpose, we replace $\left(1 / \tau_{B}=v_{B} / L_{E}\right)$ in the integrals \{Eqs. (3 and 4)\} by Eq. (36), where now $\tau_{B}$ is a function of $P$ and $l$. The thermal conductivity is then calculated for different values of $P$ below maximum $K$ and finally, the value of $P$ which yields the best fit is chosen for each nanowire diameter sample. 


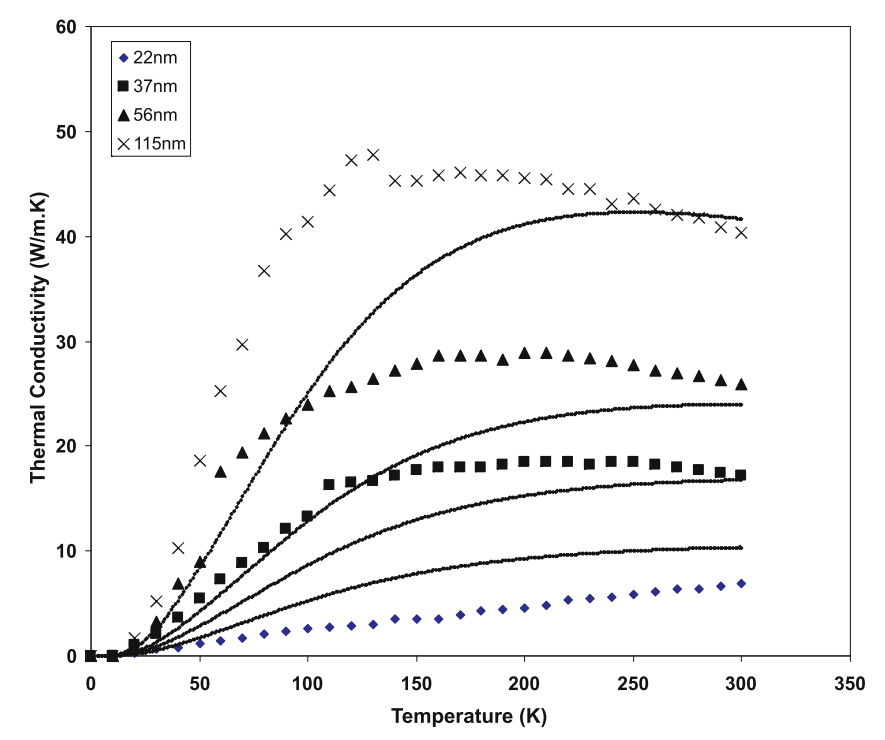

Figure 2. Temperature dependence of the calculated lattice thermal conductivity for Si nanowire using the effects of boundary scattering with the modified group velocity in comparison with that of the experimental curve taken from Li (2002).

It is well-known that when a bulk single crystal is reduced to a $1 \mathrm{D}$ which is a nanowire, then, the lattices forming the wires cross-section area are deviated from their periodicity. The degree of the lattice deviation is inversely proportional to the diameter of the nanowire. A large deviation is expected for smaller diameters. However, the lattice deviation acts as a dislocation from lattice periodicity point of view, and thus dislocation scattering is expected which is denoted by $N_{\text {imp }}$ as the impurity concentration in Eq. (18). The lattice volume, in this case will be position dependent. This new non periodic lattice arrangement creates an extra unharmonic lattice vibration which is represented by the Gruneisen parameter $\gamma$. In this work, attempts have been made to use all the three size dependent parameters $P, N$ and $\gamma$ to correlate the calculated lattice thermal conductivity to that of the corresponding experimental curves shown in figure 2. Throughout the method of trial and error, using the MATHCAD 12 program, the values of $P, N_{\text {imp }}$ and $\gamma$ were adjusted such that the best fit for calculated lattice thermal conductivity to the experimental curves were obtained as shown in figure 3 . The diameter dependence of the fitting parameters $N_{\text {imp }}, P$ and $\gamma(L, T)$ and $\gamma$ (total) are shown in figures $4,5,6,7$ and 8 respectively.

\section{Analysis of results}

Figure 2 is the experimental curves of lattice thermal conductivities of individual single crystalline intrinsic Si nanowires with diameters of $115,56,37$ and $22 \mathrm{~nm}$ and length of $4 \mu$ have been measured using a microfabricated suspended device over a temperature range of 20 $320 \mathrm{~K}$ ( $\mathrm{Li}$ 2002). The nanowires have been synthesized by the vapor-liquid-solid method in which Au clusters has been used as a solvent at high temperature (Li 2002). All the nanowires reported as single crystalline and were grown along the $\{111\}$ direction (Li 2002). To obtain figure 2, all parameter values used for calculating lattice thermal conductivity for bulk $\mathrm{Si}$ shown in figure 1 are also used for Si nanowires except $v_{g}$ and $\theta_{D}$, where they are recalculated values for all diameters used. A large deviation between the calculated and experimental (see figure 2) indicates the existence of other size dependent parameters such as $P, N_{\text {imp }}$ and $\gamma$ where their explanations are given in the following section. 


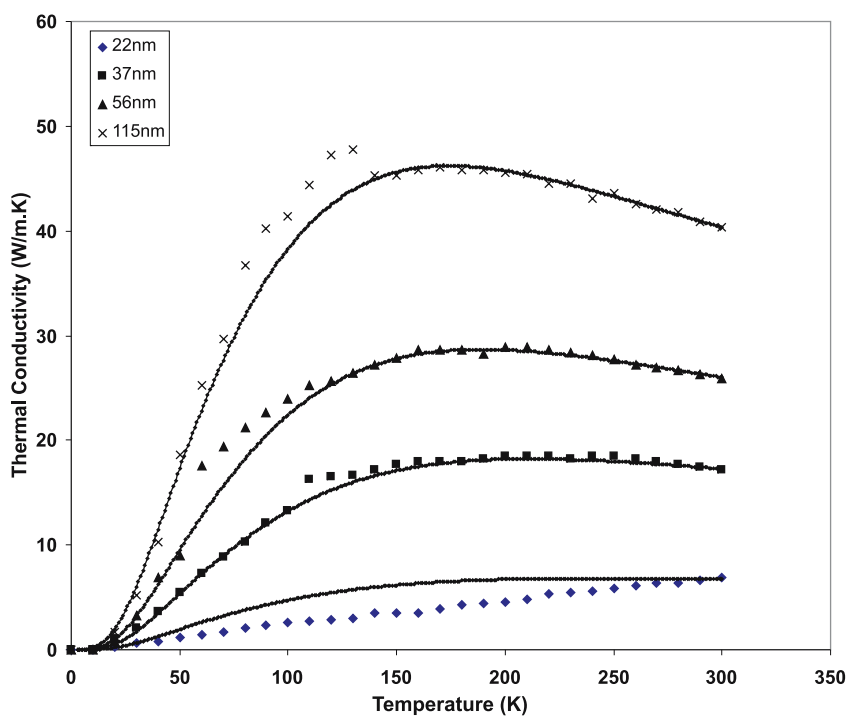

Figure 3. Temperature dependence of the calculated lattice thermal conductivity for Si nanowire using the effects of boundary scattering, modified group velocity as well as the size dependent parameters of lattice dislocation $N_{\text {imp }}$. Surface roughness $P$ and the Gruneisen parameter $\gamma$ in comparison with the experimental curve taken from Li (2002).

\subsection{Dislocations}

When a bulk crystal reduces to a nanoscale size, nanowire in this case, the lattice periodicity is unaffected in the direction of the length of the wire ( $Z$ direction for example). In the wires cross-section ( $x y$ plane), a geometrical disturbance is expected to occur. First, the periodicity of the lattice from wire cross-section center increases in a systematic form from the minimum inter-atomic bonding which is mostly for the bulk to that of the maximum bonding length at the surface. In the center of the wire cross-section the bonding length is for the bulk (like a rode) which is for Si crystal is equal to $0.235 \mathrm{~nm}$ (Omar 2007), then it increases with decreasing the wire diameter $D$, and at the surface it becomes equal to $0.667 \mathrm{~nm}$ (Zhang et al 2001). Lattice arrangements of successive circular layers at the wire cross-section will be deformed

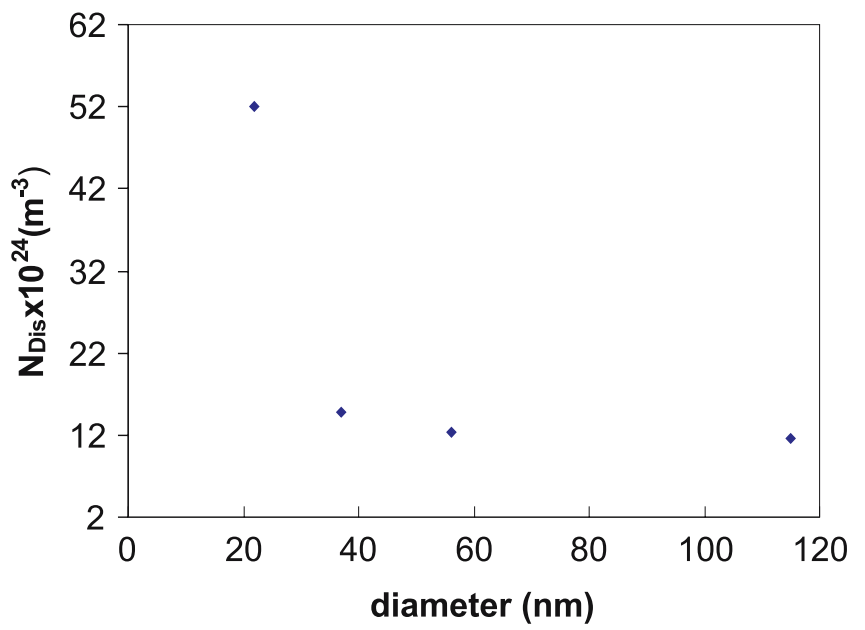

Figure 4. The impurity concentration (dislocation) versus $\mathrm{Si}$ nanowire diameter. 


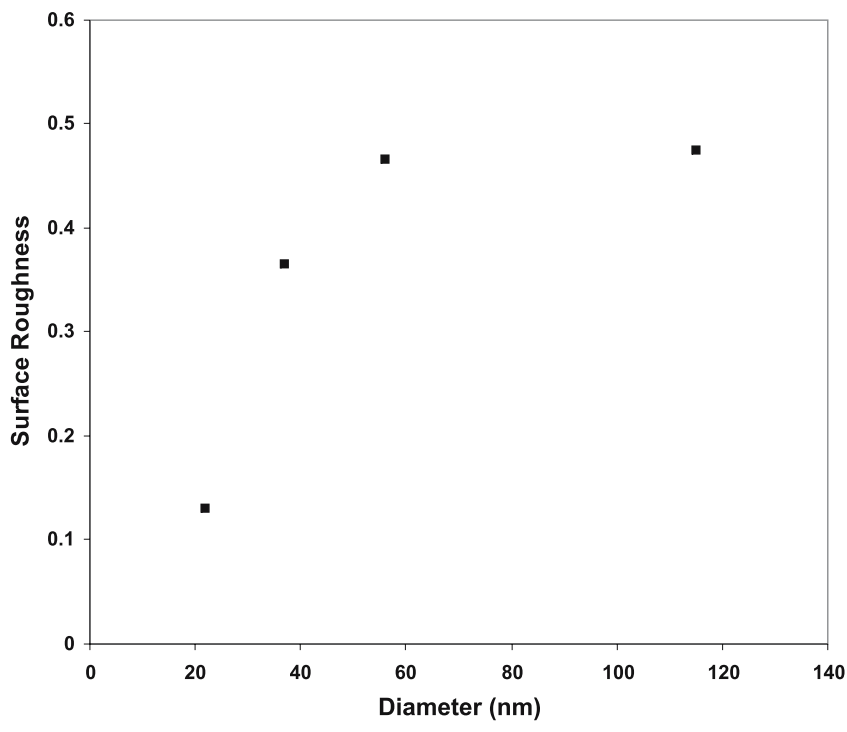

Figure 5. The surface roughness versus Si nanowire diameter.

with regard of their lattice periodicity by transferring from the expected surface plane $\{112\}$ to another which is $\{211\}$ (Li 2002; Lee \& Rudd 2007) at their truncate. However, truncate makes an intersection angle between the two planes where it increases as the wire diameter decreases. Both assumed suggestions causes increase in the degree of dislocation with the decrease of wires diameter. It is well-known that the surface thickness for a certain material is constant and is in the range of about $5 \mathrm{~nm}$. Then, the change of materials size should only be represented by the change of the bulk part of the system. According to this approach, the properties related to the surface will remain constant while the ones belong to the bulk

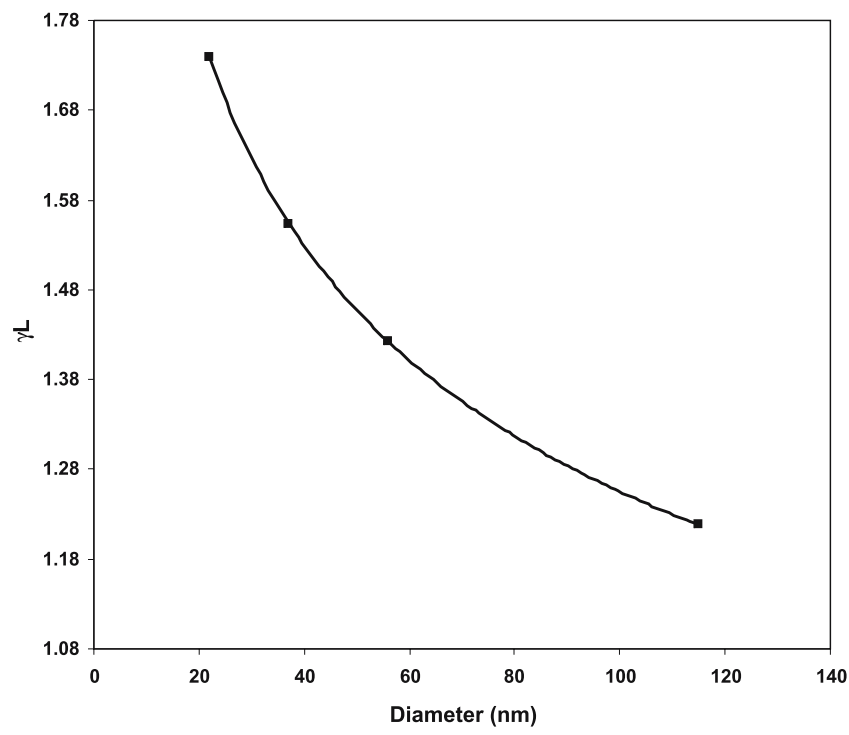

Figure 6. Longitudinal Gruneisen parameter versus Si nanowire diameter. 


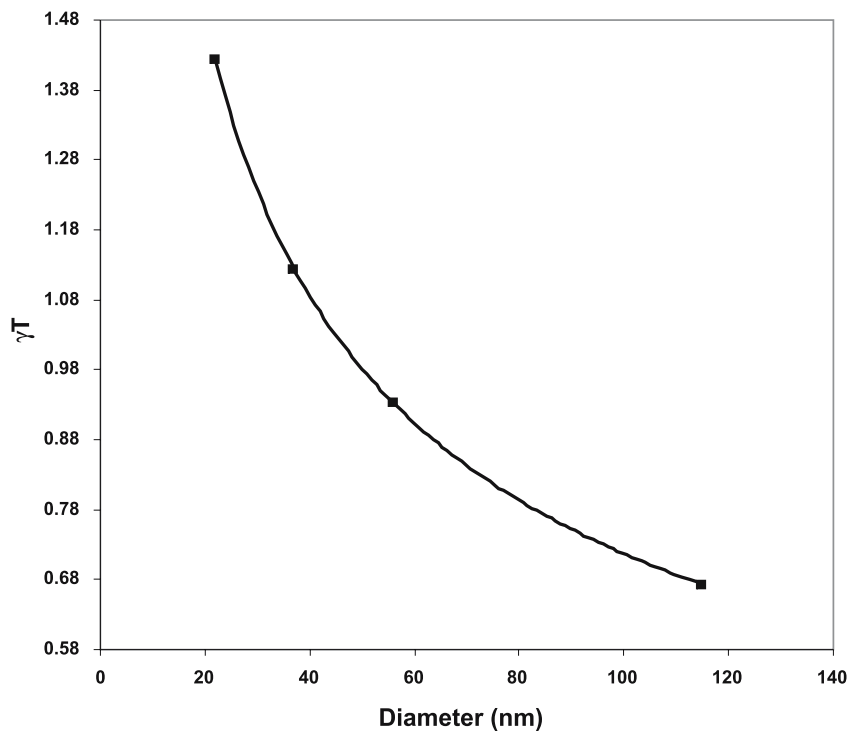

Figure 7. Transverse Gruneisen parameter versus $\mathrm{Si}$ nanowire diameter.

change in accordance to its percentage surface to bulk ratio. In this case, for a large diameter nanowire, the bulk properties are controlling the dependence of $N_{\text {imp }}$ versus $D$ curve down to the diameter $37 \mathrm{~nm}$ as shown in figure 4 . For a diameter below $37 \mathrm{~nm}$, the $22 \mathrm{~nm}$ in this work, the bulk participation is small which give a large surface to bulk ratio and then in this case, the surface effect will control the physical properties of the wire. The concentration of lattice dislocation found to change from $1.16 \times 10^{19} \mathrm{~cm}^{-3}$ for $115 \mathrm{~nm}$ to $5.20 \times 10^{19} \mathrm{~cm}^{-3}$ for the $22 \mathrm{~nm}$ wire diameter.

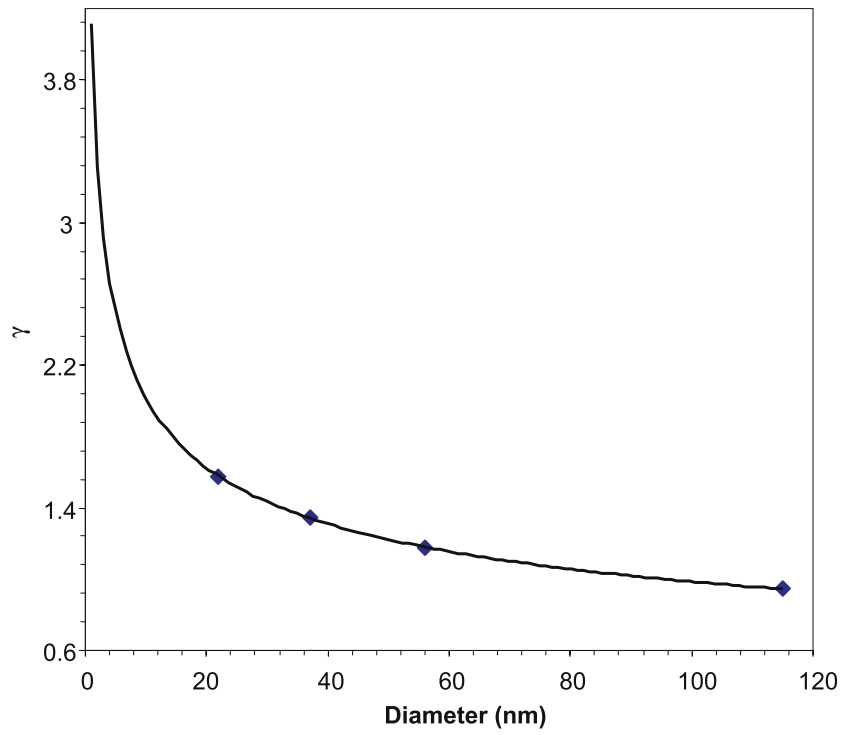

Figure 8. Total Gruneisen parameter versus Si nanowire diameter and the extension to approaching zero diameter according to the empirical relation obtained from the dependence. 


\subsection{Surface roughness}

The important of surface preparation on the magnitude of $K(T)$ near and below $K_{\max }$ for bulk crystals has been investigated in some details (Asen-Palmer et al 1997). The theory of Ziman (1956) and Sofer (1967) enables researchers to describe the influence of various degree of surface polishing on the temperature dependence of $K(T)$ in samples with a given geometry and mass. However, the specularity factor $P$, is sufficient to account for effects resulting from the surface roughness. It is well-known that the enhancement of $K$ due to specular reflection is considerably larger at lower temperature than the higher ones. It is also known that the higher the boundary limited thermal conductivity, the lower the temperature below which $T^{3}$ law is valid. For the case of nanoscale size materials such as nanowires, this phenomenon controls $K(T)$ for temperatures much higher than that of the bulk.

If the sample surface assumed to have specular reflectivity or $P=1$ before the surface has been bent (for the bulk case), then for a nanowire, the bend assumption model which is mentioned in the lattice dislocation explanation, is applicable to define the state of the surface. Figure 5 shows roughness versus diameter dependence for the four diameters 115, 56, 37 and $22 \mathrm{~nm}$ giving roughness values in the range of $0.475 \geq P \geq 0.13$. In highly polished samples the problem of phonon reflections are possible to be described according to the explanation given by Ziman (1960) and Sofer (1967). They gave the specularity factor dependence on the phonon wave vector $\vec{k}$ to be as

$$
P(\vec{k})=\exp \left[-(2 \vec{k} \eta \cos \Phi)^{2}\right],
$$

where $\eta$ is the rms height deviation in the surface and $\Phi$ is the angle of phonon incidence, since the temperature gradient is along the length of the sample. For a specular reflection from a plane surface, $\eta=0$ and then $P(\vec{k})=1$ regardless of values of $\vec{k}$ and $\Phi$. Since the temperature gradient along the length of the sample expected to be the same for bulk and nanowire, the effects of $\Phi$ and $\vec{k}$ in Eq. (36), are also expected to be the same as long as both are a function of temperature, and their values should be $\Phi<90^{\circ}$ and $\vec{k}>0$.

According to this equation, values of $P$ should represent the rms height deviation $\eta$ from the nanowire surface. In this case, as values of $P$ decrease, values of $\eta$ increase in accordance. This assumption can be explained as follows. According to the assumption made by (Lee \& Rudd 2007), the nanowire surface is constructed from intersection of crystal plains forming an internal angle between them such as $\beta$. For a large nanometer diameter, number of planes forming wires surface are large and then their internal angles are also large, while for a small diameter nanowire, the number of these planes are smaller and produces a smaller internal angles. If we plot a circle tangent to the internal surface planes, then the difference between the circle radius and the head of the angle is the rms deviation from the wires surface. However, smaller internal angles will give a higher rms deviation from the wires surface, which is represented by $\eta$. For bulk state $(D=\infty), \beta$ is $180^{\circ}$, and that gives $\eta=0$ which represents a specular reflection as originally assumed for this case in the present work. However, $\beta=90^{\circ}$ for a nanowire having $D=1.39 \mathrm{~nm}$ (Lee \& Rudd 2007) where in this case the circle assumption above give $\eta \approx \frac{1}{3} D$. According to Eq. (37), the shape of the curve indicated in figure 5, will give an increased values of $\eta 1.66$ when the nanowire diameter goes down from $115 \mathrm{~nm}$ to $22 \mathrm{~nm}$.

\subsection{Gruneisen parameter}

When a crystal size reduces to a nanoscale range, the surface to bulk ratio will begin to dominate the mechanical properties of the material. It is well-known that the surface bonding 
length is much larger than bonds belong to the bulk material (Zhang et al 2001). As mentioned before, the mean bonding length for a nanoscale range material increases according to the increase of surface to bulk ratio. For a nanowire shape, as in the present work, the diameter can be regarded as a parameter to describe the materials structure. In this case, the decrease of the diameter $D$ should correspond to the increase of mean bonding length and consequently it means the increase of the volume per atom. Such a conclusion can be used to correlate the Gruneisen parameter $\gamma$ versus $D$ diagram (see figure 8). Since the inter-atomic force in crystals are usually weak for large lattice displacements, the Gruneisen parameter will have positive values for all phonon modes, which means proportional to the crystal lattice volume $V$. For such assumption the increase of lattice volume should consequently be a function of the wire diameter $D$. The best fit dependence of $\gamma$ versus $D$ in figure 8 , gives the empirical relation of $\gamma=5 \cdot 1076 D^{-0 \cdot 3927}$ ( $D$ in nm). According to this relation, a strong control of surface effect will take place at diameters down from 10 to $12 \mathrm{~nm}$ as shown in figure 8 , which means a surface thickness of about 5 to $6 \mathrm{~nm}$. However, a similar surface thickness has been reported for thin films of metrahedral amorphous carbon (Liu et al 1999).

Lee \& Rudd (2007) and (Omino et al 2005) reported the decrease of young's modulus in the direction of crystal planes $\{001\}$ and $\{110\}$ as the wires diameter decease with respect to that of the bulk Young's modulus, while for the direction belongs to the crystal plane $\{111\}$ the dependence is opposite to that. For both cases, the dependence on wire diameter will become more sensitive as the wire diameter decreases. However, for most binary compounds from groups III-V and II-VI, the Gruneisen parameter has been reported to increase as a function of Bulk modulus (Iwanaga et al 2000). Then, for certain nanowire diameters, the increase of bulk modulus should correspond to the increase of $\gamma$. Since in general, the bulk modulus is equal to three times of that of Young's modulus, $\gamma$ should have the same proportional dependence on Young's modulus. In this case, the diameter dependence of $\gamma$ (see figure 8) has the same shape as that for the dependence reported for Young's modulus versus Si nanowires diameter along the crystal plane $\{111\}$ (Lee \& Rudd 2007).

In general, Young's modulus is dependent on the planes forming the wires surface. Since the direction of the length for Si nanowires investigated in this work is $\{111\}$ (Li 2002), the planes forming the wires surface are expected be $\{112\}$ and $\{211\}$ and their truncations. Therefore, the dependence mentioned above and the information from reference (Liang et al 2005), the Gruneisen parameter dependence on diameter in this work should correspond to the nanowire surface plane.

\section{Conclusions}

The temperature dependence of lattice thermal conductivity for the nanowire diameters from 115 to $22 \mathrm{~nm}$ indicates that the system used in the present calculation is perfectly applicable to all Si nanowire diameters down to $37 \mathrm{~nm}$ while it partially works for the $22 \mathrm{~nm}$ diameter wire. The dependence of surface roughness and defects on the wire diameter indicate a strong control of surface in the surface to bulk ratio for the $22 \mathrm{~nm}$ Si nanowire diameter. Results of the three parameters $P, N_{\text {imp }}$ and $\gamma$ in the correlation between theoretical and experimental curves of lattice thermal conductivity in nanowires for all the diameters presented in this work indicate the significance of size dependent parameters. 
This work is a part of a PhD research project supervised by Dr M S Omar at the Department of Physics, College of Science, University of Salahaddin in Arbil, Iraqi Kurdistan, Iraq. The facility and the financial support are acknowledged.

\section{References}

Asen-Palmer M, Bartkowski K, Gmelin E, Cardona M, Zhernov A P, Inyushkin A V, Taldenkov, Ozhogin V I, Itoh K M, Haller E E 1997 Thermal conductivity of germanium crystals with different isotopic compositions. Phys. Rev. B56: 9431-9447

Balandin A, Wang K L 1998 Significant decrease of the lattice thermal conductivity due to phonon confinement in a free-standing semiconductor quantum well. Phys. Rev. B58: 1544-1549

Berman R, Simon F E, Ziman J M 1953 The thermal conductivity of Diamond at low temperatures. Proc. R. Soc. London, Ser. A 220: 171-183

Berman R 1976 Thermal conductivity in solids (Oxford: Oxford University Press)

Callaway J 1959 Model for lattice thermal conductivity at low temperature. Phys. Rev. 113: 1046-1051

Casimir H B G 1938 Note on the conduction of heat in crystals. Physica 5: 495-500

Chen Ke-Qiu, Li Wen-Xia, Duan Wenhui, Shuai Z, Gu Bing-Lin 2005 Effect of defect on the thermal conductivity in a nanowire. Phys. Rev. B72: 045422-045426

Dash J G 1999 History of the search for continuous melting. Rev. Mod. Phys. 71: 1737-743

Dolling G, Cowley R A 1966 The thermodynamic and optical properties of germanium, silicon, diamond and gallium arsenide. Proc. Phys. Soc. London 88: 463-494

Herring C 1954 Role of low-energy phonons in thermal conduction. Phys. Rev. 95: 954-965

Holand M G 1963 Analysis of lattice thermal conductivity. Phys. Rev. 132: 2461-2471

Iwanaga H, Kunishige A, Takeuchi S 2000 Anisotropic thermal expansion in wurtzite type crystals. J. Mat. Sci. 35: 2451-2454

Klemens P G 1955 Thermal conductivity and lattice vibrational modes. Proc. R. Soc. London, Ser. A 68: $1113-1121$

Lee B, Rudd R E 2007 First-principles study of the Young's modulus of Si $\langle 001\rangle$ nanowires. Phys. Rev. B75: 041305-041308(R)

Li D Y 2002 Thermal transport in individual nanowire and nanotube. Ph.D. Thesis, (Berkeley: Univ. of California)

Li D Y, Wu Y, Fan R, Yang P D, Majumdar A 2003 Thermal conductivity of individual silicon nanowires. Appl. Phys. Lett. 83: 3186-3188

Liang L H, Baowen Li 2006 Size-dependent thermal conductivity of nanoscale semiconducting systems. Phys. Rev. B73(15): 153303-153306

Liang H, Upmanyu M, Huang H 2005 Size-dependent elasticity of nanowires: Nonlinear effects. Phys. Rev. B71: 241403-241406(R)

Lindemann F A 1910 The calculation of molecular vibration frequency. Z. Phys. 11: 609-612

Liu E, Shi X, Tan H S, Cheah L K, Sun Z, Tay B K, Shi J R 1999 Surface and Coatings Technol. 120-121, 601-606

Lu X, Shen W Z, Chu J H 2002 Size effect on the thermal conductivity of nanowres. J. Appl. Phys. 91(3): 1542-1552

Mahan G, Sales B, Sharp J 1997 Thermoelectric materials: New approaches to an old problem. Phys. Today 50(1): 42-47

Mingo N 2003 Calculation of nanowire thermal conductivity using complete phonon dispersion relations. Phys. Rev. B68: 113308-113311

Morelli D, Heremans J P, Slack G A 2002 Estimation of the isotope effect on the lattice thermal conductivity of group IV and group III-V semiconductors. Phys. Rev. B66: 195304-195312

Murphy P G, Moore J E 2007 Coherent phonon scattering effects on thermal transport in thin semiconductor nanowires. Phys. Rev. B76: 155313-155323

Omar M S 2007 Lattice thermal expansion for normal tetrahedral compound semiconductors. Materials Res. Bull. 42: 319-326 
Post E J 1953 On the characteristic temperatures of single crystals and the dispersion of the Debye heat wave. Can. J. Phys. 31: 112-119

Regel A R, Glazov V M 1995 Entropy of melting of semiconductors. Semiconductors 29(5): 405-417

Santamore D H, Cross M C 2001 Effect of phonon scattering by surface roughness on the universal thermal conductance. Phys. Rev. Let. 87: 115502-115505

Slack G A, Galginaitis S 1964 Thermal conductivity and phonon scattering by magnetic impurities in CdTe. Phys. Rev. 133: A253-A268

Soffer S B 1964 Statistical model for the size effect in electrical conduction. J. Appl. Phys. 38: 1710-1715

Thacher P D 1967 Effect of boundaries and isotopes on the thermal conductivity of LiF. Phys. Rev. 156: 975-988

Umeno Y, Kuskima A, Kitamura T, Gumbsch P, Ju Li 2005 ab initio study of the surface properties and ideal strength of (100) silicon films. Phys. Rev. B72: 165431-165437

Vandersande J W 1977 Low temperature thermal conductivity of two natural Diamond; Anisotropic heat conduction in the scattering regim. Phys. Rev. B15: 2355-2362

Wen Z, Zhao M, Jiang Q J 2000 The melting temperature of molecular nanocrystals at the lower bound of the mesoscopic size range. Phys. Condens. Matter 12: 8819-8824

Xu C H, Wang C Z,Chan C T, Ho K M 1991 Theory of the thermal expansion of Si and Diamond. Phys. Rev. B43: 5024-5027

Zhang Z, Zhao M, Jiang Q 2001 Melting temperature of semiconductor nanocrystals in the mesoscopic size range. Semicond. Sci. Technol. 16: L33-L35

Ziman J M 1956 The general vibrational principle of transport theory. Can. J. Phys. 34: 1256-1273

Ziman J M 1960 Electrons and Phonons (London: Oxford University) p. 456 